\title{
Cancer Biology and Treatment
}

\section{Role of H-Ferritin in Radio- sensitivity of Human Glioma Cells}

\section{Min Pang, Xiaoli Liu, Becky Slagle-Webb, Achuthamangalam B Madhankumar and James R Connor*}

Department of Neurosurgery, Milton S Hershey Medical Center, Pennsylvania State University College of Medicine, Hershey, USA

\section{Abstract}

Human gliomas are notorious for their resistance to conventional therapy and high recurrence, thus there is a need for improvement in therapeutic strategies. Human glioma cells have modified iron metabolism, as well as an elevated level of the iron storage protein, ferritin. Previously we have shown that the down-regulation of one of the ferritin subunits, $\mathrm{H}$-ferritin, increases sensitivity of human glioma cells to the chemotherapeutic agent $\mathrm{BCNU}$. Here we test the hypothesis that $\mathrm{H}$-ferritin down-regulation increases radiosensitivity in glioma cells and explore the mechanism for the sensitization effect. Our data demonstrated enhanced radiosensitivity in glioma cells transfected with $\mathrm{H}$-ferritin siRNA both in vitro and in vivo. Further investigation revealed that in our cell culture model, $\mathrm{H}$-ferritin down-regulation is accompanied with a disruption in iron metabolism, increased oxidative damage, and decreased stability of Hypoxia-Inducible Factor (HIF)-2a. Additionally, we discovered that in the presence of ionizing radiation, down-regulation of $\mathrm{H}$-ferritin was associated with decreased level of the phosphorylated form of ATM kinase. Our present study supports a role for $\mathrm{H}$-ferritin in tumor growth and maintenance, identifies $\mathrm{H}$-ferritin as a target in cancer cells. Importantly, we demonstrate that we have a nanotechnology platform for delivery of siRNA in vivo. Keywords: Glioma; H-ferritin; Iron metabolism; Oxidative stress; Radiosensitization
\end{abstract}

\section{Introduction}

Glioma is the most common type of malignant brain tumor. The highest grade (grade IV) glioma, Glioblastoma Multiforme (GBM), is characterized by high morbidity, mainly due to its rapid growth, resistance to therapy, and high relapse rate. Clearly, there is a significant unmet clinical need for the improvement of glioma

*Corresponding author: James R Connor, Department of Neurosurgery, Milton S Hershey Medical Center, Pennsylvania State University College of Medicine, Hershey, USA, Tel: +1 7175314541; Fax: +1 7175310091; E-mail: jconnor@psu.edu

Citation: Pang M, Liu X, Slagle-Webb B, Madhankumar AB, Connor JR (2016) Role of H-Ferritin in Radiosensitivity of Human Glioma Cells. J Cancer Biol Treat 3: 006.

Received: December 14, 2015; Accepted: January 07, 2016; Published: January 21, 2016 treatment. A number of studies have been published seeking molecular targets within the cancer cells to either destroy them or sensitize them to increased efficacy of existing therapies. Our recent publication has shown that in a subcutaneous glioma mouse model, the therapeutic effect of Bis-Chloroethyl Nitrosourea (BCNU or carmustine) can be enhanced when combined with the down-regulation of $\mathrm{H}$-ferritin, but not L-ferritin [1]. However, the treatment of choice for many brain tumors is radiotherapy. Thus, we expanded our investigation to the role of $\mathrm{H}$-ferritin in radiosensitivity of glioma and the mechanism by which reduced expression of ferritin increases sensitivity to radiation.

Ferritin is the major iron storage protein in mammalian cells. It is essential to sequester iron and thereby protect cells from oxidative damage induced by excess iron. Ferritin is distributed in all cells and typically is found as a complex composed of 24 subunits. These complexes consist of two different gene products: $\mathrm{H}$-ferritin and L-ferritin. Although they share a multi-helical three-dimensional structure and a homology of 50-56\% in amino acid sequences, their functions are distinctive $[2,3]$. The $\mathrm{H}$-ferritin subunit has ferroxidase and anti-oxidant activity, which converts ferrous iron into ferric iron. L-ferritin modifies the microenvironment and facilitates iron uptake through nucleation. The iron uptake process is accomplished by the cooperation of both $\mathrm{H}$ - and L- subunits [4]. The subunit composition varies based on the cellular iron requirement of the tissue: $\mathrm{H}$-subunit is abundant in brain and heart, while L- subunit is enriched in liver and spleen [5].

In addition to the cytoprotective role of intracellular ferritin through direct iron management, both $\mathrm{H}$ - and L-ferritin genes contain an Antioxidant/Electrophilic Responsive Element (ARE/ EpRE) upstream from their initiation sites. Thus there is evidence for the cytoprotective role of ferritin in response to oxidative stress at the gene expression level [6]. Ferritin induction by oxidative stress and the consequential cytoprotection has been identified in various systems. For example, over expression of either $\mathrm{H}$ - or L-ferritin subunits can reduce the accumulation of ROS in hela cells [7]. In addition the H-ferritin subunit has been shown to bind to DNA and may function to promote transcription through controlled release of iron that can relax DNA [8].

The relationship between iron and cancer has been investigated for decades. The carcinogenic effect of iron is attributed to iron accumulation in association with oxidative stress induction and subsequent DNA damage [9]. The most egregious example of this relationship is hereditary hemochromatosis, an iron overload disorder that is identified as an enhanced risk factor for hepatocellular carcinoma [10]. Iron-induced oxidative stress can generate selected oncogenic mutations and increase cell proliferation, both of which are carcinogenic in a cooperative way [11]. In cancer cells, the iron requirement is thought to be increased due to their rapid growth and elevated metabolism. To meet the high iron demand, transferrin receptor, a major component of the route for cellular iron intake, is up-regulated in many types of cancer cells [12]. In brain tumors, both forms of transferrin receptors, TfR1 and TfR2 are up-regulated compared with normal brain tissue $[13,14]$ and supports 
using the transferrin receptor as a mechanism to gain entry of anti-cancer agents into cancer cells $[15,16]$. Elevated iron levels in both serum and tissue from tumor-bearing individuals are observed [9]. The robust iron uptake by cancer cells must be regulated to keep the cells viable and this concept supports a rationale for our approach targeting iron management proteins, such as ferritin, [1] in cancer treatment.

The present study investigates the potential role of $\mathrm{H}$-ferritin siRNA, delivered through a liposomal delivery system, as an adjuvant therapy for increasing sensitivity of tumors to therapeutic strategies. The results we present provide first evidence that the radiosensitivity of human glioma cells can be enhanced by targeting ferritin, the iron storage protein.

\section{Materials and Methods}

\section{Cell culture}

Human glioma cell line U251 was purchased from American Type Culture Collection. The cells were maintained in DMEM (GIBCO) supplemented with 10\% FBS (v/v), 1\% (v/v) Non-Essential Amino Acid Solution (Sigma), and 0.1\% (v/v) Gentamicin Reagent Solution (GIBCO). Cells were maintained in a humidified atmosphere of $5 \%$ $\mathrm{CO}_{2} / 95 \%$ air at $37^{\circ} \mathrm{C}$. The cumulative culture duration of all cells was less than 6 months.

\section{H-ferritin down-regulation in vitro}

$\mathrm{H}$-ferritin down-regulation was performed by liposomal transfection of $\mathrm{H}$-ferritin siRNA. Liposomes in the size range of 50-100 nm were formulated using DC-cholesterol: DOPE lipid and characterized as described in our previous report and complexed with $\mathrm{H}$-ferritin siRNA for transfection [1]. Briefly, DC-cholesterol: DOPE lipid (Avanti) was solubilized in 1:1 (v/v) chloroform: methanol mixture and rotary evaporated to form a thin film followed by reconstitution in Phosphate Buffered Saline (PBS) and extrusion in a lipex extruder (Northern Lipids Inc.) utilizing 50 and $100 \mathrm{~nm}$ membranes, both for 10 times. At transfection, siRNA was complexed with liposomes in the ratio of 1:4 (siRNA $(\mu \mathrm{g})$ : liposomes $(\mu \mathrm{L}))$ at room temperature for 10 minutes. The liposomes were originally positively charged and were neutralized after being complexed with siRNA. The liposomes containing H-ferritin siRNA (Santa Cruz) or a non-specific siRNA (Qiagen) were added to U251 cells at approximately $60 \%$ confluency in serum free medium. Three hours later, the medium was aspirated and complete culture medium was applied. The cells were collected at 24, 48, and 72 hours post transfection and protein expression was analyzed to determine knockdown efficiency.

\section{Radiosensitization by $\mathrm{H}$-ferritin siRNA transfection in vitro and terminal deoxynucleotidyl Transferase dUTP Nick End Labeling (TUNEL) assay}

U251 cells transfected with $\mathrm{H}$-ferritin siRNA or non-specific siRNA underwent $2,5,10$, or 20 Gy of ionizing radiation at 24 hours post transfection. Ionizing radiation treatment was performed by a Co-60 gamma irradiator (Nordion), using the empty chamber method. One-solution MTS cell proliferation assay (Promega) was performed at 24, 48, and 72 hour post radiation. Another set of irradiated cells were fixed with $4 \%$ paraformaldehyde at 1 hour or 24 hours post radiation and TUNEL assay (Promega) was performed following the manufacturer's instructions.

\section{Radiosensitization by $\mathrm{H}$-ferritin siRNA transfection in vivo}

Ten adult female athymic nude mice (Charles Rivers Laboratories) were implanted in the flank subcutaneously with $1.5 \times 10^{7} \mathrm{U} 251$ cells. When the tumors reached $40-50 \mathrm{~mm}^{3}$, mice were randomly assigned to receive injections of either (a) liposomes: non-specific siRNA or (b) liposomes: $\mathrm{H}$-ferritin siRNA $(6 \mu \mathrm{L} / 1.5 \mu \mathrm{g})$. In both cases complexed liposomes in a total volume of $50 \mu \mathrm{L}$ were injected intratumorally on a weekly basis. We have previously shown that this dosing regimen will decrease ferritin expression in the tumors [1]. Mice were exposed to $4 \mathrm{~Gy}$ of whole body $\gamma$-radiation two days after liposomal injection. This regimen was repeated for 7 weeks with a cumulative dose of 28 Gy. The radiation dose was chosen based on the literature standard [17-19]. Tumor volume was recorded weekly by an individual blinded to the treatment strategy. No untreated control mice were used for humane reasons. The studies were approved by Institutional Animal Care and Use Committee at Pennsylvania State University College of Medicine.

\section{Protein immunoblot}

After cells were collected, lysis buffer containing RIPA buffer, protease inhibitor cocktail, and phosphatase inhibitor cocktail (all from Sigma) was used for protein extraction. Samples were sonicated on ice and centrifuged to remove cell debris. Protein concentration was then determined using the BCA protein assay kit (Pierce). SDS-PAGE was performed in criterion precast gels and proteins were transferred to nitrocellulose or PVDF membrane (Life Sciences) in criterion blotter (Bio-rad) at $4^{\circ} \mathrm{C}$. After transfer, the blots were incubated in blocking solution, $5 \%$ non-fat milk or BSA in Tris-Buffered Saline with 0.05\% Tween-20 (TBST), for 1 hour. The blots then underwent overnight incubation with primary antibody dissolved in $5 \%$ non-fat milk or BSA in TBST at $4^{\circ} \mathrm{C}$. Specifically, anti-H-ferritin, anti-pATM (pS1981), anti-ATM, anti-actinin (Cell Signaling Technology), anti-transferrin receptor (Invitrogen), anti-HIF-2 $\alpha$, anti-IRP2 (Novus Biologicals), anti- $\alpha$-actin and anti- $\beta$-actin (Sigma) primary antibodies were used. After 3 washes in TBST, the blots were incubated in HRP-conjugated secondary antibodies for mouse or rabbit (GE healthcare) dissolved in 5\% non-fat milk for 1 hour at room temperature. After another 3 washes, the blots were incubated in Western Lightning PLUS substrate (Perkin Elmer) for 1 minute and exposed. Multi Gauge software was used for image quantification.

\section{Calcein AM assay}

At 24,48 , and 72 hours post transfection of non-specific siRNA or $\mathrm{H}$-ferritin siRNA, calcein AM (Sigma) was added to the cells in serum free medium (to a final concentration of $0.5 \mu \mathrm{M}$ ) for 30 minutes at $37^{\circ} \mathrm{C}$. The cells were washed with PBS twice, and the fluorescent signal was quantified using a fluorescent plate reader (spectramax Gemini, Molecular Devices), with excitation wavelength at $495 \mathrm{~nm}$ and emission wavelength at $515 \mathrm{~nm}$.

\section{Iron assay}

At 24, 48, and 72 hours post transfection, cells were collected in PBS. Iron content in these cells was quantified using the Iron Assay Kit (Abcam). Briefly, cells were lysed in assay buffer. Ferrous iron in the samples reacted with Ferene $S$ and resulted in a colorimetric product. Then the optical density at $593 \mathrm{~nm}$ of the samples was analyzed by a plate reader (Spectramax 340PC384, Molecular Devices, LLC). The readings were normalized to their respective protein 
concentrations that were obtained using the BCA protein assay (Pierce). Measurements were taken in duplicates.

\section{Protein oxidation assay}

Protein oxidation was measured with oxyblot protein oxidation detection kit (Millipore) and the OxiSelect ${ }^{\mathrm{TM}}$ protein carbonyl ELISA kit (cell biolabs) following the manufacturer's instructions. Protein oxidation levels from the cell lysate samples were normalized based on the protein concentrations obtained from a BCA protein assay. Measurements were taken in triplicates.

\section{Supercoil relaxation assay}

Covalently closed circular pUC19 DNA plasmids (biolabs) were utilized, as in our previous report [20]. One $\mu$ g plasmid DNA was dissolved in $10 \mathrm{mM}$ Hepes ( $\mathrm{pH} 7.5$ ), $50 \mathrm{mM} \mathrm{NaCl}, 2.5 \mathrm{mM} \mathrm{MgCl}$, and $2.5 \mathrm{mM}$ DTT (total volume of $30 \mu \mathrm{L}$ ). The plasmid and reaction mixture was incubated in the presence or absence of $\mathrm{H}$ - or L-ferritin for 1 hour at $37^{\circ} \mathrm{C}$, and the reactions were terminated with $50 \%(\mathrm{v} / \mathrm{v})$ glycerol, 50mM EDTA and $0.1 \%$ bromophenol blue. The samples were exposed to $\gamma$-radiation and then loaded on an agarose gel (1.5\%) and electrophoresed at $40 \mathrm{~mA}$ for 8 hours at room temperature. After electrophoresis, the gel was immersed in $100 \mathrm{ml}$ of TAE buffer containing $5 \mu \mathrm{L}$ of SYBR Safe DNA gel stain (Invitrogen) for 10 minutes. The conversion of relaxation, linear and supercoiled forms of DNA was examined using a LAS 3000 Fuji image reader. Recombinant $\mathrm{H}$-ferritin expressed and purified in our laboratory following our earlier method [21] and horse spleen ferritin (Sigma) were used as L-ferritin.

\section{Statistical analysis}

Results are shown as mean \pm SEM. Unless otherwise noted, a one-way ANOVA and Tukey's post hoc test was used for statistical analysis. A value of $\mathrm{p}<0.05$ is considered as statistically significant.

\section{Results}

\section{H-ferritin siRNA enhances radiation efficacy both in vitro and in vivo}

A cell proliferation assay revealed that the transfection of H-ferritin siRNA sensitized U251 glioma cells to high doses of radiation (10 and $20 \mathrm{~Gy}$, Figure 1a). To elucidate the mechanism of this inhibitory effect on cell proliferation, TUNEL assay was performed to determine the apoptosis level in cells treated with $\mathrm{H}$-ferritin siRNA transfection and $20 \mathrm{~Gy}$ of radiation. While no significant difference was observed at 1 hour post radiation, the percentage of TUNEL-positive cells was significantly increased in cells with $\mathrm{H}$-ferritin siRNA transfection at 24 hours post radiation (Figure $1 \mathrm{~b}$ ). Next, we tested the radiosensitization effect of $\mathrm{H}$-ferritin siRNA in a mouse subcutaneous glioma model. Tumor growth was effectively suppressed by 100 fold at week 5 and 1,000 fold at week 7 in the group of mice receiving weekly intratumoral injections of liposomes carrying $\mathrm{H}$-ferritin siRNA and whole body radiation at $4 \mathrm{~Gy}$ compared to mice receiving non-specific siRNA injection and radiation (Figure 1c). The study was terminated at week 7 . The $\mathrm{H}$-ferritin siRNA transfection group was observed for another 3 weeks for potential tumor regrowth and the tumors did not recur.

$\mathrm{H}$-ferritin down-regulation affects iron metabolism in human glioma cells. To further investigate the underlying mechanism for the sensitization effect, we studied the iron metabolism in glioma cells with $\mathrm{H}$-ferritin siRNA transfection. We hypothesized that $\mathrm{H}$-ferritin

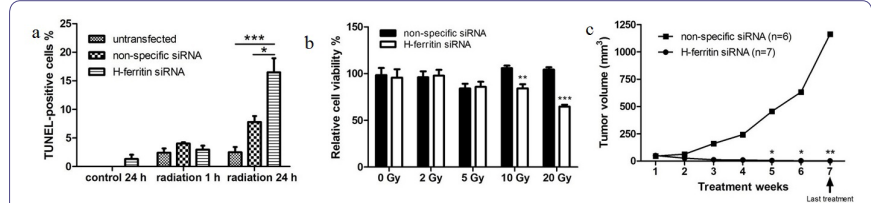

Figure 1: H-ferritin siRNA transfection enhances both in vitro and in vivo radiosensitivity of glioma cells.

(a) Glioma cells treated with H-ferritin siRNA are sensitized to high doses of radiation. Cells were transfected with liposomes containing either non-specific siRNA or $\mathrm{H}$-ferritin siRNA and followed with radiation of various doses at 24 hours post transfection. MTS cell proliferation assays were performed after another 24 hours. Cells treated with 10 and 20 Gy of radiation showed significantly decreased cell proliferation rate. Cell proliferation rates in untransfected cells are set at $100 \%$ as the control. Error bars represent SEM $\left({ }^{* *}, p<0.01 ;{ }^{* * *}, p<0.001\right)$.

(b) Apoptosis is activated in cells with $\mathrm{H}$-ferritin siRNA transfection and $20 \mathrm{~Gy}$ of radiation, represented by increased percentage of TUNEL-positive staining at 24 hours post radiation. Error bars represent SEM $\left({ }^{*}, p<0.05 ;{ }^{* *}, p<0.01\right)$.

(c) H-ferritin siRNA injection enhances the efficacy of radiation in a subcutaneous mouse model of glioma cells. The average tumor size in mice treated with weekly injection of $\mathrm{H}$-ferritin siRNA followed by $4 \mathrm{~Gy}$ of whole body radiation 48 hours later was significantly reduced starting from week 5 , when compared with those treated with non-specific siRNA injections and radiation. Error bars represent \pm SEM $\left({ }^{*}, p<0.05 ;{ }^{* *}, p<0.01\right)$. The animals receiving siRNA injections were allowed to survive for three weeks after radiation treatment ended (denoted by the arrow) during which time no tumor growth was observed.

down-regulation will affect intracellular iron metabolism. Following exposure to $\mathrm{H}$-ferritin siRNA, decreases in $\mathrm{H}$-ferritin expression were observed in U251 cells at 24, 48, and 72 hours post transfection compared to untransfected cells and cells with non-specific siRNA transfection (Figure 2a). We also monitored Transferrin Receptor (TfR) expression because it is responsive to the intracellular iron status [22]. TfR level was decreased at 48 hours post transfection and was similar to control levels at 72 hours post transfection, suggesting a transient decrease in iron intake in cells with $\mathrm{H}$-ferritin siRNA transfection. The analysis of IRP2, the member of IRP family whose expression is post-translationally regulated by heme-dependent ubiquitination [23], revealed a decrease of $79 \%$ and $36 \%$ in cells with $\mathrm{H}$-ferritin siRNA transfection at 48 and 72 hours post transfection, respectively (Figure 2b).

To more directly assess the effect of $\mathrm{H}$-ferritin down-regulation on iron metabolism, a calcein AM assay was performed (Figure 2c). Calcein $\mathrm{AM}$ is a fluorescent probe that can penetrate the cell membrane and its fluorescent signal is quenched by divalent metals, with the highest affinity in ferrous iron [24]. Ferrous iron that is unbound and reactive in the cells is called the 'Labile Iron Pool' (LIP) and the level of LIP is negatively correlated with fluorescent signal in calcein AM assay. The results showed that at 24 and 48 hours post transfection, no significant difference was observed. However, at 72 hours post transfection, the fluorescent signal in cells with $\mathrm{H}$-ferritin down-regulation was significantly increased, suggesting a decreased level of LIP. In addition, an iron assay was performed to confirm the results shown above. This colorimetric assay is designed to quantify the unbound ferrous iron that forms the LIP. The results showed that at 48 hours post transfection, the iron level in cells with $\mathrm{H}$-ferritin down-regulation was decreased by $43 \%$, in comparison with untransfected cells (Figure 2c). These data both indicate that cells with $\mathrm{H}$-ferritin down-regulation have less biologically reactive cytoplasmic iron. 


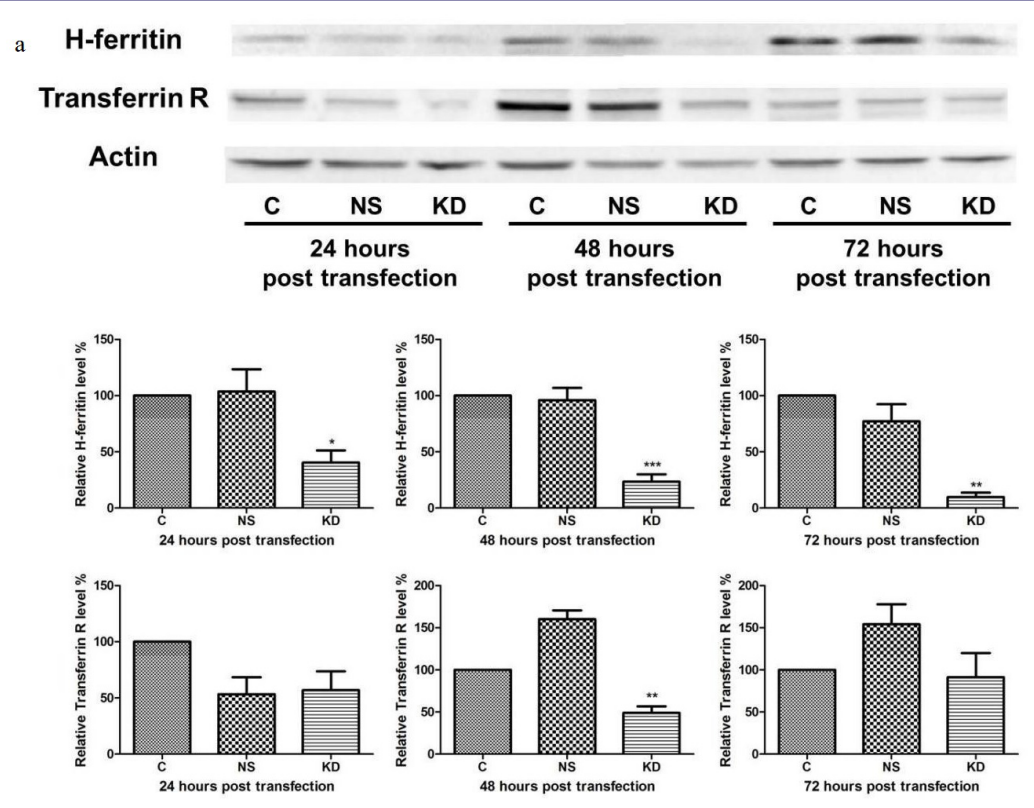

b
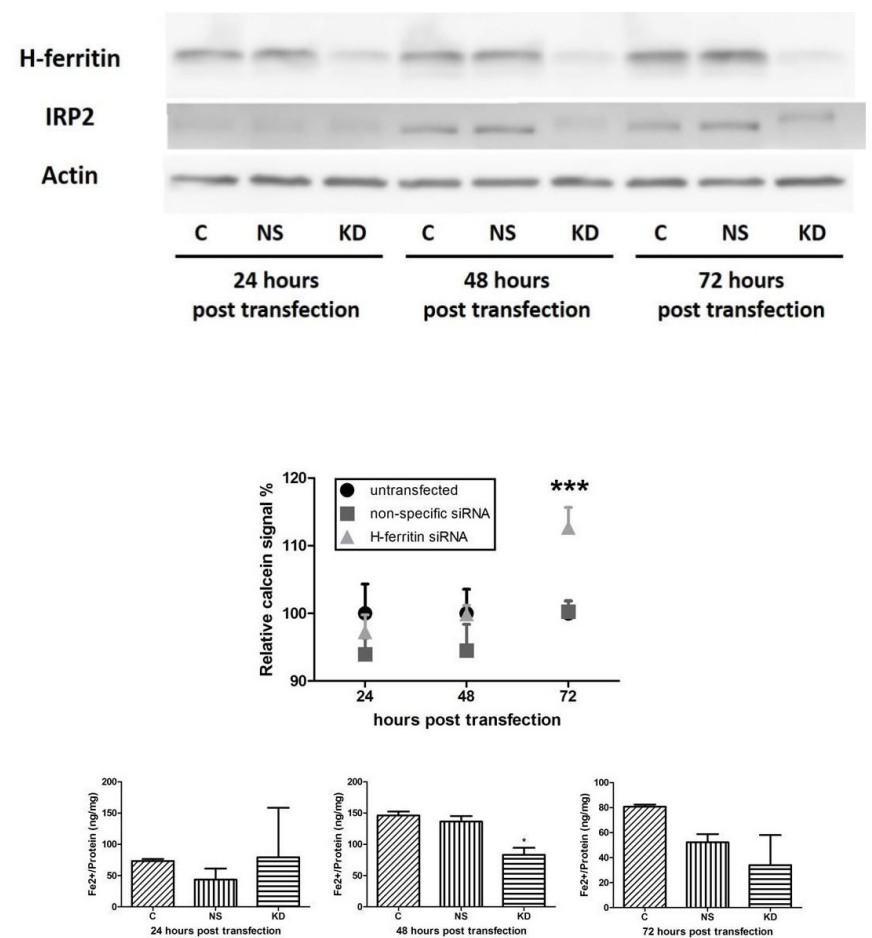

Figure 2: H-ferritin down-regulation affects iron metabolism in glioma cells.

(a) In glioma cells with liposomal transfection of $\mathrm{H}$-ferritin siRNA, $\mathrm{H}$-ferritin down-regulation is accompanied with a transient decrease of transferrin receptor (Transferrin R) level at 48 hours post transfection. Top: A representative protein immunoblot. Middle: A summarized quantification of five protein immunoblots for $\mathrm{H}$-ferritin. Bottom: A summarized quantification of four protein immunoblots for transferrin receptor. Relative protein levels in untransfected cells at each time point are set for $100 \%$ control. Error bars represent SEM (C: Untransfected cells; NS: cells transfected with non-specific siRNA; KD: cells transfected with H-ferritin siRNA. **, $p<0.01)$.

(b) H-ferritin down-regulation leads to a decreased level of IRP2 with a shift in molecular weight at both 48 and 72 hours post transfection. (C: Untransfected cells; NS: cells transfected with non-specific siRNA; KD: cells transfected with $\mathrm{H}$-ferritin siRNA.)

(c) Cells with $\mathrm{H}$-ferritin siRNA transfection exhibit a decreased level of labile iron pool, demonstrated by calcein AM assay and iron assay. Top: calcein AM assay showed a significantly elevated fluorescent signal in cells with $\mathrm{H}$-ferritin siRNA transfection at 72 hours post transfection, suggesting a decreased level of labile iron pool in such cells. Bottom: In an iron assay, a transient decrease in ferrous iron content was observed in cells with $\mathrm{H}$-ferritin siRNA transfection at 48 hours post transfection. Error bars represent SEM (C: Untransfected cells; NS: cells transfected with non-specific siRNA; KD: cells transfected with H-ferritin siRNA. *, p<0.05; $* * *, p<0.001)$. 


\section{$\mathrm{H}$-ferritin down-regulation is associated with an increase in intracellular oxidative stress level}

Fenton reaction describes a process that converts unbound ferrous iron to ferric iron, while produces hydroxyl radicals [25]. Therefore, the loss of effective iron management may lead to the generation of oxidative stress. U251 cells transfected with H-ferritin siRNA exhibited elevated protein oxidation starting at 48 hours post transfection and returned to normal level by 72 hours (Figure 3a). This transient induction of protein oxidation suggests that $\mathrm{H}$-ferritin down-regulation not only disrupts iron metabolism, but also oxidative homeostasis. To further investigate the cytotoxic effect of $\mathrm{H}$-ferritin down-regulation in combination with radiation, the level of protein oxidation was determined in cells treated with both H-ferritin siRNA transfection and radiation. The results showed that the combination of siRNA and 20 Gy of radiation resulted in an additive effect on protein oxidation (Figure $3 b$ ). However, no significant difference was observed in cells treated with lower doses of radiation $(2,5$, and 10 Gy).
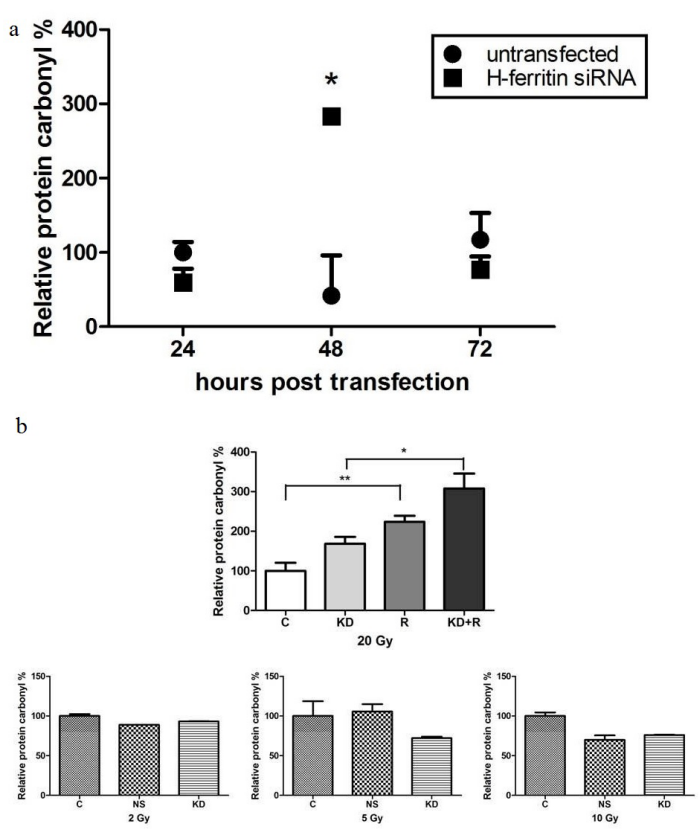

HIF-2 $\alpha$
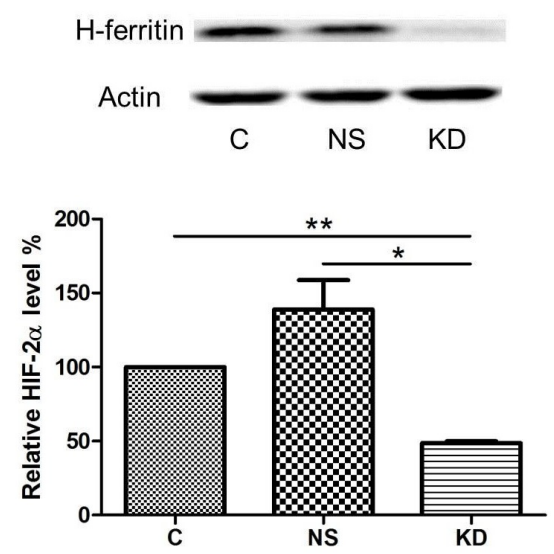

Figure 3: H-ferritin down-regulation affects oxidative homeostasis and hypoxia in glioma cells.

(a) $\mathrm{H}$-ferritin down-regulation transiently increases the level of protein oxidation damage compared to untreated control cells at 48 hours post transfection. Error bars represent SEM $\left({ }^{*}, p<0.05\right)$.

(b) $\mathrm{H}$-ferritin down-regulation exacerbates the oxidative damage induced by radiation. Top: cells treated with high dose of radiation (20 Gy) exhibited elevated level of protein oxidation damage, while $\mathrm{H}$-ferritin down-regulation had an additive effect. Error bars represent SEM. (C: Untransfected cells. KD: Cells transfected with $\mathrm{H}$-ferritin siRNA. R: Cells treated with radiation. KD+R: Cells treated with radiation 48 hours post $\mathrm{H}$-ferritin siRNA transfection. The data were analyzed for statistical differences using two-way ANOVA and Tukey post hoc analysis. ${ }^{*}, p<0.05 ;{ }^{* *}, p<0.01$.) Bottom: no significant effect in protein oxidation was observed with lower doses of radiation (2, 5, or $10 \mathrm{~Gy}$ ). Error bars represent SEM (C: untransfected cells. NS: cells transfected with non-specific siRNA. KD: cells transfected with $\mathrm{H}$-ferritin siRNA).

(c) $\mathrm{H}$-ferritin down-regulation destabilizes HIF-2 $\alpha$ in glioma cells. Top: In cells with H-ferritin siRNA transfection, HIF-2 $\alpha$ level is decreased at 72 hours post transfection. A representative protein immunoblot is shown. Bottom: Quantification of three protein immunoblots, normalized to respective actin levels. Error bars represent SEM (C: untransfected cells. NS: cells transfected with non-specific siRNA. KD: cells transfected with $\mathrm{H}$-ferritin siRNA. ${ }^{*}, p<0.05 ;{ }^{* *}, \mathrm{p}<0.01$ ). 


\section{The stability of hypoxia-inducible factor is decreased in cells with $\mathrm{H}$-ferritin siRNA transfection}

Hypoxia-Inducible Factors (HIFs) are gene regulators that are accumulated under hypoxic conditions in mammalian cells. The hypoxic status and level of HIFs in cancer may be crucial in the therapeutic efficacy of anti-cancer treatments [26]. Specifically, HIF- $2 \alpha$ has been linked with glioma cell survival and glioma stem cell maintenance and renewal $[27,28]$. Here we observed a decreased level of HIF- $2 \alpha$ in cells with $\mathrm{H}$-ferritin siRNA transfection at 72 hours post transfection (Figure 3c).

\section{Discussion}

We predicted that the disruption of one link of the delicate feedback loop, namely ferritin, in cells for maintaining iron homeostasis and cytoprotection, could have therapeutic significance in cancer. The idea for the analysis of $\mathrm{H}$-ferritin in cancer cells began with the observations that $\mathrm{H}$-ferritin knockout mice were embryonic lethal $[30,31]$ and that $\mathrm{H}$-ferritin was found in the nucleus of dividing cells [20]. These studies strongly suggested a critical role for ferritin in early cell development. In order to apply this knowledge to cancer biology and tumor growth we developed a nanotechnology platform to

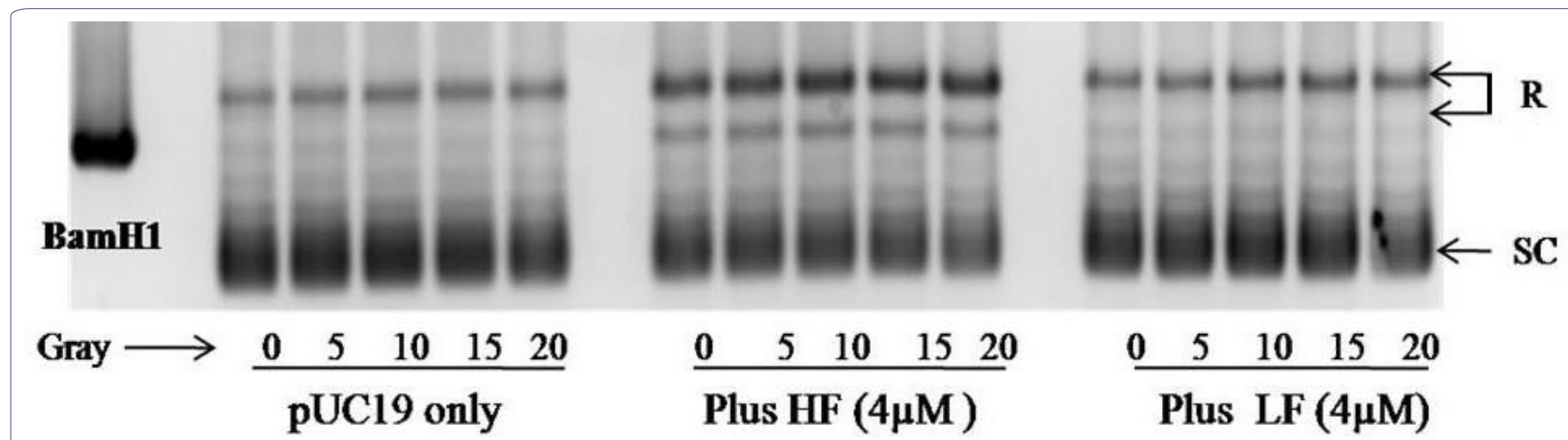

Figure 4: Influence of $\mathrm{H}$-ferritin on DNA relaxation studied by a supercoil relaxation assay.

DNA plasmid pUC19 was treated with $4 \mu \mathrm{M}$ of $\mathrm{H}$-ferritin (plus HF) or L-ferritin (plus LF) and then exposed to incremental concentrations of radiation. The conversion to Relaxed $(\mathrm{R})$ forms was observed in the presence of $\mathrm{rH}$-ferritin but remarkably less in the presence of L-ferritin. Radiation had a minimal effect. (SC: Supercoiled form.)

\section{H-ferritin maintains pUC19 DNA plasmid in a relaxed and} linear form even under radiation

The DNA plasmid pUC19 is found in either relaxed or supercoiled form. The DNA supercoil relaxation assay revealed that exposing the pUC19 DNA plasmid to radiation had minimal effect on its appearance. The presence of $\mathrm{H}$-ferritin was associated with a dramatic increase in the relaxed form, which is not affected by radiation. This effect was specific to $\mathrm{H}$-ferritin as demonstrated by the presence of L-ferritin which had no effect on the appearance of the DNA in the presence or absence of radiation (Figure 4).

The sustained activity of the DNA damage repair mechanism induced by radiation is impaired by $\mathrm{H}$-ferritin down-regulation

One of the mechanisms of action in radiation induced cell death is the generation of DNA damage, mainly double strand breaks. However, some glioma cells demonstrate enhanced DNA repair activity upon radiation, which leads to radio resistance [29]. In this study, we tested the phosphorylation level of ATM protein (pS1981), which is the key kinase that recruits and activates a cascade of DNA repair and checkpoint proteins once the double strand break is detected. The results showed no pATM was observed in cells without radiation (Figure 5). At 1 hour post radiation, significant pATM was induced in all cells regardless of transfection or non-transfection. At 24 hours post radiation, significantly lower levels of both pATM and ATM were observed in cells with $\mathrm{H}$-ferritin siRNA transfection. This result suggests that pATM formation can be induced by radiation as early as 1 hour post radiation. The transfection of $\mathrm{H}$-ferritin siRNA decreased this PATM level at a later time point, presumably through the down-regulation of ATM expression. produce an acute decrease in ferritin expression. Using this approach we previously demonstrated that a significant but transient change in iron homeostasis in cancer cells following exposure to ferritin siRNA is coupled with an increased sensitivity to chemotherapeutic agent [1] and we expand this finding in the present study to radiosensitivity in both in vitro and in vivo models. The results of this study demonstrate that reducing expression of $\mathrm{H}$-ferritin in human glioblastoma cells disrupts iron homeostasis, promotes oxidative stress, alters DNA repair mechanisms, and increases cell sensitivity to radiation. The data are also consistent with reports that the persistent depletion of ferritin in normal cells may be deleterious [30-32].

In the first set of experiment, we determined the in vitro and in vivo radiosensitivity of glioma cells with $\mathrm{H}$-ferritin siRNA transfection. In our transfection system, neither the liposomes nor the transient down-regulation of $\mathrm{H}$-ferritin inhibits glioma cell growth or exhibits cytotoxicity, as demonstrated by the observation that $\mathrm{H}$-ferritin siRNA transfection showed no significant inhibition effect on proliferation without radiation (Figure 1). However, the cell proliferation rates are significantly reduced in cells with $\mathrm{H}$-ferritin siRNA transfection by high doses of radiation (10 and $20 \mathrm{~Gy}$ ), suggesting a radiosensitization effect (Figure 1a). Further analysis revealed that this growth inhibition effect is due to an induction in apoptosis activity (Figure 1b). In addition, our in vivo study demonstrated a significant tumor suppression effect by $\mathrm{H}$-ferritin down-regulation with a relatively low dose of radiation (4 Gy per week, Figure 1c).

This dose preference in sensitization suggests that the disruption caused by $\mathrm{H}$-ferritin down-regulation in the in vitro study may not be strong enough to produce a sensitization effect at lower doses of radiation. Meanwhile, in the mouse model, multiple treatments apparently results in accumulation of disruptive events that result in the significant sensitization effect observed. Therefore further studies 


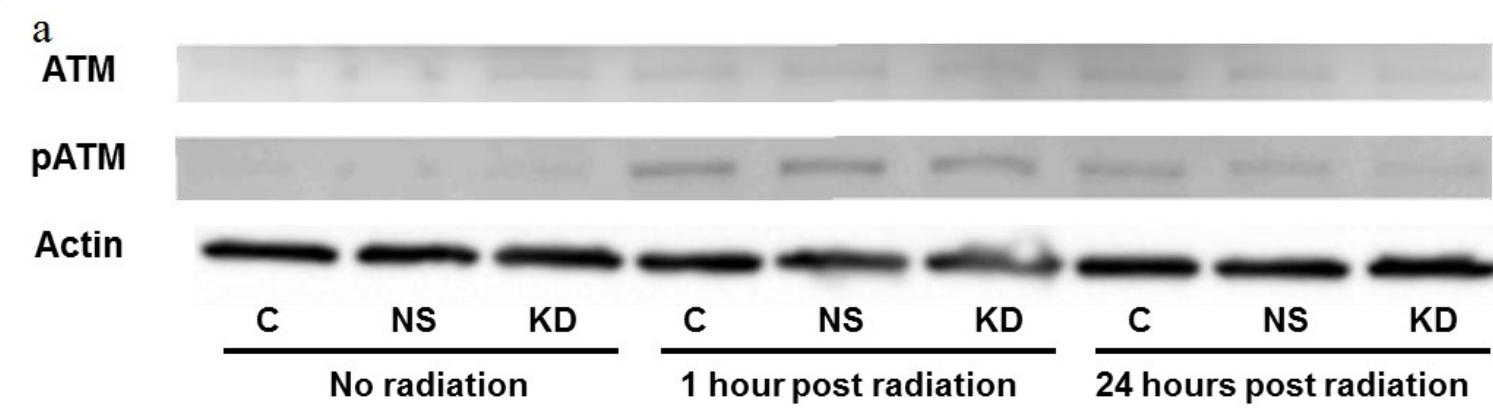

b
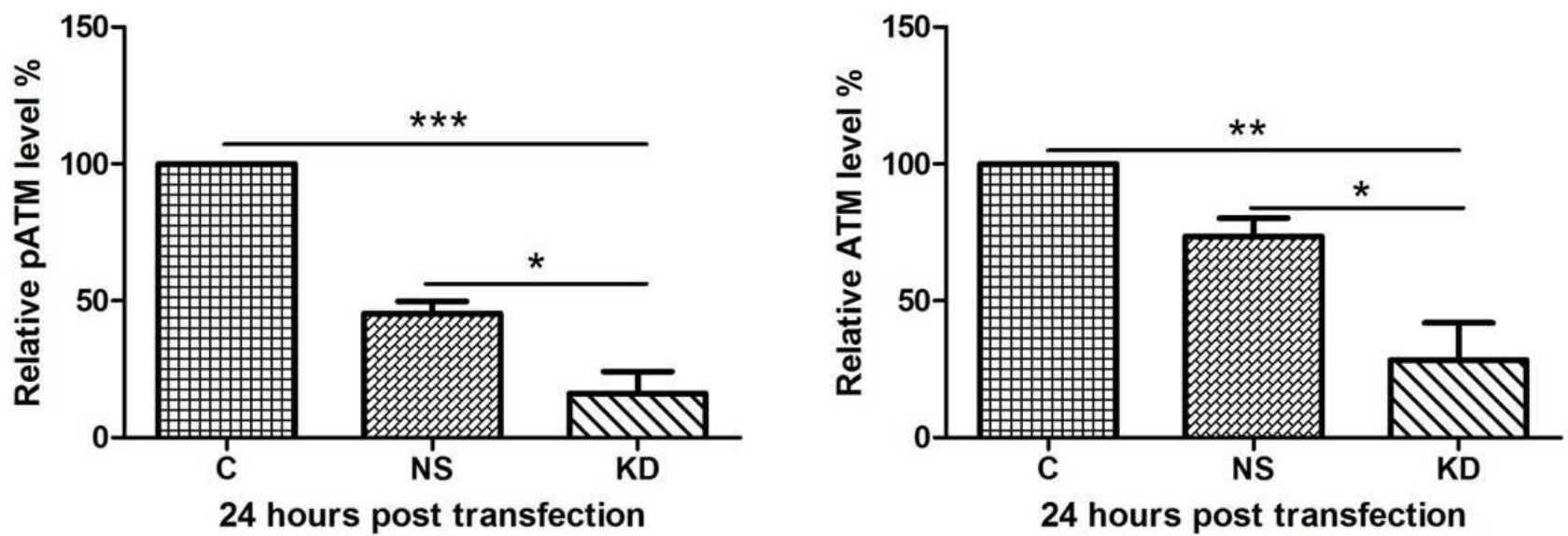

Figure 5: The activity of the DNA damage response protein ATM is impaired in cells with $\mathrm{H}$-ferritin down-regulation.

(a) Levels of ATM, pATM (pS1981), and actin in cells treated with H-ferritin siRNA and 20 Gy of radiation. No pATM level was observed in cells without radiation treatment. At 1 hour post radiation, pATM was induced in both transfected and non-transfected cells. By 24 hours post radiation, pATM is significantly reduced in the cells with the greatest reduction occurring in those receiving $\mathrm{H}$-ferritin siRNA transfection. Total ATM levels were also decreased at 24 hours in the cells that were transfected with siRNA for $\mathrm{H}$-ferritin.

(b) Quantification of three protein immunoblots. Levels of pATM and ATM at 24 hours post radiation are normalized to respective actin or actinin levels. Error bars represent SEM (C: cells without transfection. NS: cells transfected with non-specific siRNA. KD: cells transfected with $\mathrm{H}$-ferritin siRNA. *, p<0.05; ${ }^{* *}$, p<0.01; ${ }^{* * *}$, $p<0.001$ ).

were proposed to investigate the underlying mechanisms for this sensitization effect.

Our investigation on iron metabolism suggests that the effects of $\mathrm{H}$-ferritin siRNA transfection occur in two phases. In the first phase (0-48 hours post transfection), $\mathrm{H}$-ferritin down-regulation is accompanied with a decrease in the level of transferrin receptor and IRP2 (Figure 2a and b). To support normal cell growth, ferritin is constitutively degraded and iron that is previously captured in the ferritin cage is released. The transfection of $\mathrm{H}$-ferritin siRNA blocks the re-synthesis of $\mathrm{H}$-ferritin to scavenge such iron, which is likely to result in an accumulation of iron compounds such as heme and Fe-S clusters. As a result, both IRP2 and transferrin receptor are down-regulated (Figure 2a and $b$ ). In the second phase (48-72 hours post transfection), the depletion of $\mathrm{H}$-ferritin may cause a reduced rate of ferritin degradation and a lower level of subsequent iron release, which is reflected by the decreased level of LIP observed at later time points post transfection (Figure 2c). Consequently, the accumulation of iron compounds is reversed and the transiently disrupted iron metabolism is partially restored at 72 hours post transfection (Figure 2a and b). This scenario accounts for both the decrease in transferrin receptor and IRP2.
A major cellular function of ferritin is to regulate iron that could be available to participate in the production of reactive oxygen species [33]. The level of oxidative stress in cancer cells is critical because free radicals induced by radiation may damage DNA and lead to subsequent cell death [34]. Indeed, compounds that are effective in producing oxidative stress and enhancing ROS generation have been reported as radio sensitizers [35-37]. In this study, we found that $\mathrm{H}$-ferritin down-regulation is associated with increased oxidative stress level in glioma cells as determined by analysis of oxidatively modified proteins (Figure 3a). High dose of radiation increases protein oxidative damage and this damage was exacerbated in cells with both $\mathrm{H}$-ferritin down-regulation and radiation (Figure $3 \mathrm{~b}$ ). The induction of oxidative damage is likely due to the lack of iron scavenging activity after $\mathrm{H}$-ferritin down-regulation. This finding also indicates that the elevation of intracellular oxidative stress may be involved as one of the mechanisms for the sensitization effect preferentially at high doses of radiation. The intricate series of event subsequent to $\mathrm{H}$-ferritin siRNA transfection is summarized in a schematic plot (Figure 6). The transient disruption in cellular metabolism is likely to provide a specific time window for the sensitization effect. 
a

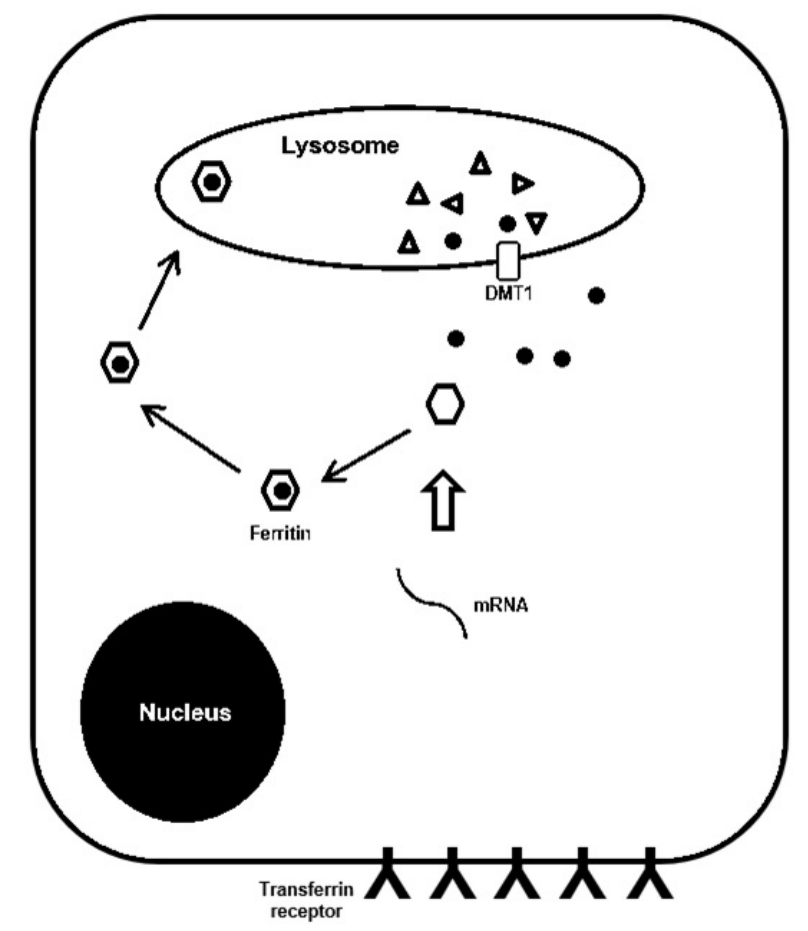

b

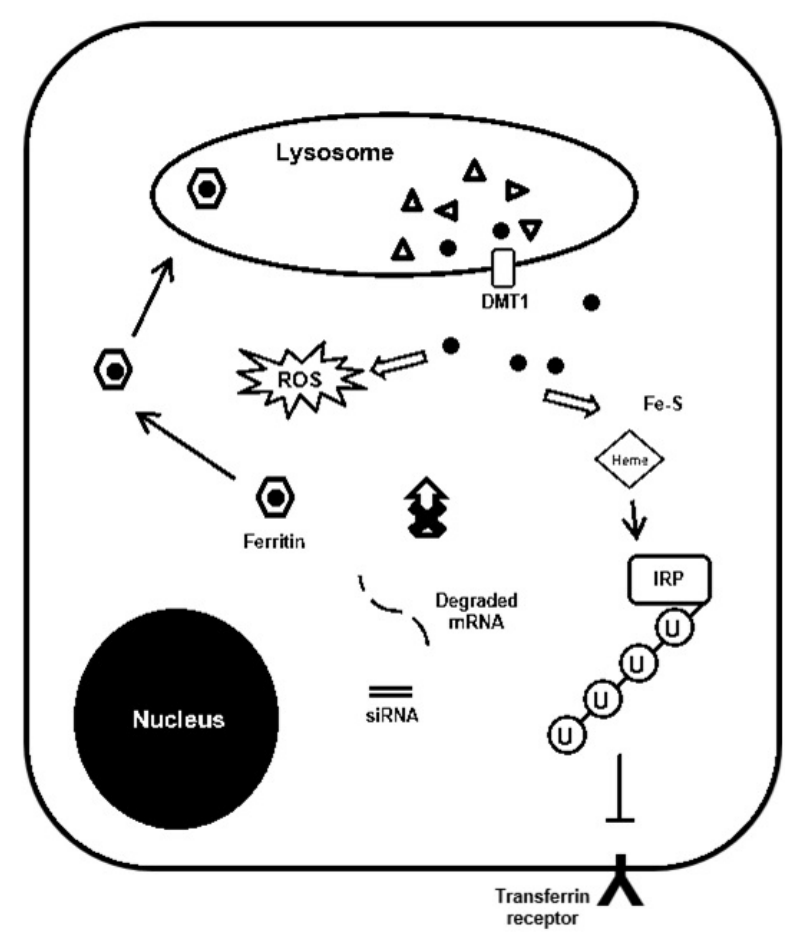

Figure 6: A schematic summarizing the effect of $\mathrm{H}$-ferritin down-regulation in glioma cells.

(a) Iron metabolism in cells without $\mathrm{H}$-ferritin siRNA transfection. Holoferritin is degraded in lysosome and iron is released to the cytosol through transporters such as DMT1. H-ferritin mRNA is translated into protein and ferritin cage is formed to recycle iron released from lysosome. Redox reaction and ROS production is prevented as a result.

(b) With the presence of siRNA, the re-synthesis of $\mathrm{H}$-ferritin is inhibited and ROS is produced by iron released from lysosome. Free iron is then captured in forms of iron-containing complexes

Cellular iron status is also known to affect the level of HIFs. HIFs are transcription factors that regulate protein expression as responses to hypoxia. Hypoxia and increased HIFs expression are well known factors promoting cancer cell resistance to radiation. The relationship between HIFs level and cancer progression has been investigated in many cancer types, and has even raised the prospect of targeting HIFs as a possible cancer therapy [26]. Specifically, HIF-2 $\alpha$ is thought to serve as a crucial factor in glioma cell survival and a prognostic marker in glioma patients $[27,38]$. Our data reveal that the HIF-2 $\alpha$ level in glioma cells is decreased after down-regulation of $\mathrm{H}$-ferritin (Figure 3c).

The intracellular level of HIFs is regulated by Prolyl Hydroxylases (PHDs), whose primary function is to hydroxylate and destabilize HIFs. The activity of PHDs is dependent both on oxygen and iron as co-factors and is inhibited by iron chelation [39]. Our observation that $\mathrm{H}$-ferritin down-regulation destabilizes HIF-2 $\alpha$ suggests PHDs may be activated. This activation may be mediated by an increased iron delivery to PHDs by the PCBP protein, which was identified as iron chaperones for both ferritin and PHDs [40,41]. It is expected that in the absence of ferritin, more iron is delivered to PHDs.

In addition to its traditional role of ferritin in the cytosol, the physiological significance of nuclear-localized ferritin has been discovered recently. Nuclear ferritin is composed mainly of $\mathrm{H}$-ferritin and is believed participate in the transcriptional regulation and DNA protection [42]. In our previous study, we found that $\mathrm{H}$-ferritin is capable of protecting naked DNA in the presence of BCNU, a DNA alkylating chemotherapeutic agent [1]. In the current study we extend this idea to radiation (Figure 4). H-ferritin increases the relaxed form of DNA, which would be associated with an increase in transcription activity. Thus, under conditions when ferritin is present in the nucleus in cancer cells, transcription could continue unabated in the presence of radiation including production of cytoprotective and reparative mechanisms in the cells.

The possibility that ferritin protects cells and allows for activation of DNA repair mechanisms was also interrogated in this study. Bao and colleagues revealed that in CD133-positive glioma stem cells, the ATM kinase is preferentially activated and confers radio resistance in glioma stem cells [29]. A recent publication also reported that by the inhibition of ATM kinase, glioma cells can be radio sensitized [43]. In our study, the down-regulation of $\mathrm{H}$-ferritin is associated with decreased expression and phosphorylation levels of ATM kinase after radiation. These data indicate that when ferritin is depleted, the DNA damage repair response is less activated through ATM down-regulation. This is also presumably accompanied with impaired cell cycle arrest and enhanced DNA sensitivity. Our data are consistent with evidence that altering cellular iron status may influence ATM activity [44,45].

In summary, our study reveals the role of $\mathrm{H}$-ferritin in the radiosensitivity of human glioma cells and indicates that targeting ferritin in cancer cells offers a therapeutic opportunity. We have demonstrated that liposomal delivery of H-ferritin siRNA enhances radiosensitivity of glioma cells both in vitro and in vivo. The proposed mechanisms of action include that in glioma cells, the down-regulation of $\mathrm{H}$-ferritin is correlated with a transient disruption 
in iron homeostasis and enhances oxidative damage to proteins. The stability of HIF- $2 \alpha$ is also decreased in cells with H-ferritin siRNA transfection that further enhances radiosensitivity. The presence of ferritin has a nuclear effect as well as it also maintains DNA in a relaxed and linear form after radiation, along with the limited response for the DNA repair kinase ATM in cells exposed to both $\mathrm{H}$-ferritin siRNA and radiation. The data further suggest that there might be a specific time window for $\mathrm{H}$-ferritin siRNA transfection to optimize therapeutic effects when combined with radiotherapy and minimize the collateral damage to non-cancer tissue. Our current study supports the concept that $\mathrm{H}$-ferritin siRNA may act as a promising adjuvant gene therapy in glioma treatment. Given the elevated expression of ferritin in several other cancer cells, this approach may not be limited to glioma.

\section{Acknowledgment}

This project is funded, in part, under a grant from the Pennsylvania Department of Health using Tobacco CURE Funds. The Department specifically disclaims responsibility for any analyses, interpretations or conclusions. The project is also funded by NIH grant R01CA169117. The authors declare no conflict of interest.

\section{References}

1. Liu X, Madhankumar AB, Slagle-Webb B, Sheehan JM, Surguladze N, et al. (2011) Heavy chain ferritin siRNA delivered by cationic liposomes increases sensitivity of cancer cells to chemotherapeutic agents. Cancer Res 71: 22402249 .

2. Boyd D, Vecoli C, Belcher DM, Jain SK, Drysdale JW (1985) Structural and functional relationships of human ferritin $\mathrm{H}$ and $\mathrm{L}$ chains deduced from cDNA clones. J Biol Chem 260: 11755-11761.

3. Orino K, Watanabe K (2008) Molecular, physiological and clinical aspects of the iron storage protein ferritin. Vet J 178: 191-201.

4. Levi S, Yewdall SJ, Harrison PM, Santambrogio P, Cozzi A, et al. (1992) Evidence of $\mathrm{H}$ - and $\mathrm{L}$-chains have co-operative roles in the iron-uptake mechanism of human ferritin. Biochem J 288: 591-596.

5. Harrison PM, Arosio P (1996) The ferritins: molecular properties, iron storage function and cellular regulation. Biochim Biophys Acta 1275: 161-203.

6. Tsuji Y, Ayaki H, Whitman SP, Morrow CS, Torti SV, et al. (2000) Coordinate transcriptional and translational regulation of ferritin in response to oxidative stress. Mol Cell Biol 20: 5818-5827.

7. Orino K, Lehman L, Tsuji Y, Ayaki H, Torti SV, et al. (2001) Ferritin and the response to oxidative stress. Biochem J 357: 241-247.

8. Surguladze N, Thompson KM, Beard JL, Connor JR, Fried MG (2004) Interactions and reactions of ferritin with DNA. J Biol Chem 279: 14694-14702.

9. Connor JR, Lee SY (2010) Iron and Cancer. In: Milner JA, Romagnolo DF (eds.). Nutrition and Health: Bioactive Compounds and Cancer. Humana Press, Springer, Berlin, Germany. Pg no: 469-496.

10. Kowdley KV (2004) Iron, hemochromatosis, and hepatocellular carcinoma. Gastroenterology 127: 79-86.

11. Toyokuni S (2009) Role of iron in carcinogenesis: cancer as a ferrotoxic disease. Cancer Sci 100: 9-16.

12. Daniels TR, Delgado T, Rodriguez JA, Helguera G, Penichet ML (2006) The transferrin receptor part I: Biology and targeting with cytotoxic antibodies for the treatment of cancer. Clin Immunol 121: 144-158.

13. Recht L, Torres CO, Smith TW, Raso V, Griffin TW (1990) Transferrin receptor in normal and neoplastic brain tissue: implications for brain-tumor immunotherapy. J Neurosurg 72: 941-945.
14. Calzolari A, Oliviero I, Deaglio S, Mariani G, Biffoni M, et al. (2007) Transferrin receptor 2 is frequently expressed in human cancer cell lines. Blood Cells Mol Dis 39: 82-91.

15. Daniels TR, Bernabeu E, Rodríguez JA, Patel S, Kozman M, et al. (2012) The transferrin receptor and the targeted delivery of therapeutic agents against cancer. Biochim Biophys Acta 1820: 291-317.

16. Dufès C, Al Robaian M, Somani S (2013) Transferrin and the transferrin receptor for the targeted delivery of therapeutic agents to the brain and cancer cells. Ther Deliv 4: 629-640.

17. Mao XW, Green LM, Gridley DS (2001) Evaluation of polysaccharopeptide effects against C6 glioma in combination with radiation. Oncology 61: 243-253.

18. Nemoto K, Ogawa Y, Matsushita H, Takeda K, Takai Y, et al. (2002) Intraoperative Radiation Therapy (IORT) for previously untreated malignant gliomas. BMC Cancer 2: 1

19. Wick W, Wick A, Schulz JB, Dichgans J, Rodemann HP, et al. (2002) Prevention of irradiation-induced glioma cell invasion by temozolomide involves caspase 3 activity and cleavage of focal adhesion kinase. Cancer Res 62: 1915-1919.

20. Surguladze N, Patton S, Cozzi A, Fried MG, Connor JR (2005) Characterization of nuclear ferritin and mechanism of translocation. Biochem $\mathrm{J} 388$ : 731-740.

21. Fisher J, Devraj K, Ingram J, Slagle-Webb B, Madhankumar AB, et al. (2007) Ferritin: a novel mechanism for delivery of iron to the brain and other organs. Am J Physiol Cell Physiol 293: 641-649.

22. Theil EC (1990) Regulation of ferritin and transferrin receptor mRNAs. J Biol Chem 265: 4771-4774.

23. Ishikawa H, Kato M, Hori H, Ishimori K, Kirisako T, et al. (2005) Involvement of heme regulatory motif in heme-mediated ubiquitination and degradation of IRP2. Mol Cell 19: 171-181.

24. Epsztejn S, Kakhlon O, Glickstein H, Breuer W, Cabantchik I (1997) Fluorescence analysis of the labile iron pool of mammalian cells. Anal Biochem 248: $31-40$.

25. Fenton HJH (1894) LXXIII.-Oxidation of tartaric acid in presence of iron. J Chem Soc Trans 65: 899-910.

26. Semenza GL (2012) Hypoxia-inducible factors: mediators of cancer progression and targets for cancer therapy. Trends Pharmacol Sci 33: 207-214.

27. Li Z, Bao S, Wu Q, Wang H, Eyler C, et al. (2009) Hypoxia-inducible factors regulate tumorigenic capacity of glioma stem cells. Cancer Cell 15: 501-513.

28. Keith B, Johnson RS, Simon MC (2011) HIF1 $\alpha$ and HIF2 $\alpha$ : sibling rivalry in hypoxic tumour growth and progression. Nat Rev Cancer 12: 9-22.

29. Bao S, Wu Q, McLendon RE, Hao Y, Shi Q, et al. (2006) Glioma stem cells promote radioresistance by preferential activation of the DNA damage response. Nature 444: 756-760.

30. Ferreira C, Bucchini D, Martin ME, Levi S, Arosio P, et al. (2000) Early embryonic lethality of $\mathrm{H}$ ferritin gene deletion in mice. J Biol Chem 275: 3021-3024.

31. Thompson K, Menzies S, Muckenthaler M, Torti FM, Wood T, et al. (2003) Mouse brains deficient in $\mathrm{H}$-ferritin have normal iron concentration but a protein profile of iron deficiency and increased evidence of oxidative stress. J Neurosci Res 71: 46-63.

32. Omiya S, Hikoso S, Imanishi Y, Saito A, Yamaguchi O, et al. (2009) Downregulation of ferritin heavy chain increases labile iron pool, oxidative stress and cell death in cardiomyocytes. J Mol Cell Cardiol 46: 59-66.

33. Pham CG, Bubici C, Zazzeroni F, Papa S, Jones J, et al. (2004) Ferritin heavy chain upregulation by NF-kappaB inhibits TNFalpha-induced apoptosis by suppressing reactive oxygen species. Cell 119: 529-542.

34. Powell S, McMillan TJ (1990) DNA damage and repair following treatment with ionizing radiation. Radiother Oncol 19: 95-108.

35. Mitchell JB, Wink DA, DeGraff W, Gamson J, Keefer LK, et al. (1993) Hypoxic mammalian cell radiosensitization by nitric oxide. Cancer Res 53: 5845-5848. 
36. Kimura K, Bowen C, Spiegel S, Gelmann EP (1999) Tumor necrosis factor-alpha sensitizes prostate cancer cells to gamma-irradiation-induced apoptosis. Cancer Res 59: 1606-1614.

37. Tian J, Zhao H, Nolley R, Reese SW, Young SR, et al. (2012) Darinaparsin: solid tumor hypoxic cytotoxin and radiosensitizer. Clin Cancer Res 18: 33663376 .

38. Scrideli CA, Carlotti CG Jr, Mata JF, Neder L, Machado HR, et al. (2007) Prognostic significance of co-overexpression of the EGFR/IGFBP-2/HIF-2A genes in astrocytomas. J Neurooncol 83: 233-239.

39. Epstein AC, Gleadle JM, McNeill LA, Hewitson KS, O'Rourke J, et al. (2001) C. elegans EGL-9 and mammalian homologs define a family of dioxygenases that regulate HIF by prolyl hydroxylation. Cell 107: 43-54.

40. Shi H, Bencze KZ, Stemmler TL, Philpott CC (2008) A cytosolic iron chaperone that delivers iron to ferritin. Science 320: 1207-1210.
41. Nandal A, Ruiz JC, Subramanian P, Ghimire-Rijal S, Sinnamon RA, et al. (2011) Activation of the HIF prolyl hydroxylase by the iron chaperones PCBP1 and PCBP2. Cell Metab 14: 647-657.

42. Alkhateeb AA, Connor JR (2010) Nuclear ferritin: A new role for ferritin in cell biology. Biochim Biophys Acta 1800: 793-797.

43. Golding SE, Rosenberg E, Adams BR, Wignarajah S, Beckta JM, et al. (2012) Dynamic inhibition of ATM kinase provides a strategy for glioblastoma multiforme radiosensitization and growth control. Cell Cycle 11: 1167-1173.

44. Shackelford RE, Manuszak RP, Johnson CD, Hellrung DJ, Link CJ, et al. (2004) Iron chelators increase the resistance of Ataxia telangeictasia cells to oxidative stress. DNA Repair (Amst) 3: 1263-1272.

45. Shackelford RE, Fu Y, Manuszak RP, Brooks TC, Sequeira AP, et al. (2006) Iron chelators reduce chromosomal breaks in ataxia-telangiectasia cells. DNA Repair (Amst) 5: 1327-1336. 